\title{
Tratamiento térmico superficial del acero U8 mediante láser de $\mathrm{Nd}$ :YAG
}

\author{
J.S. Ceballos-Ruano $(*)$, G. Muñiz $(* *)$, R. Sagaró $(*)$, B. Barja ${ }^{(*)}$ y C. Torres ${ }^{(*)}$
}

Resumen Utilizando un láser continuo de Nd: YAG se procedió al endurecimiento superficial del acero U8. Se expone la metodología empleada en el trabajo para la determinación de los parámetros del proceso sobre la base de la documentación existente y a las pruebas realizadas. Se logra explicar el fenómeno observado para ilustrar los limites de operación de los parámetros del proceso a pesar de las limitaciones y simplificaciones realizadas. Se muestra que en el proceso de endurecimiento térmico superficial del acero U8 la profundidad endurecida está estrechamente vinculada con la potencia incidente $(P)$ en la zona de interacción, el diámetro del haz $(d)$ y la velocidad de barrido del haz del láser $(v)$ sobre la probeta por la relación $P / \sqrt{d v}$. El experimento fue diseñado estadísticamente y permitió obtener durezas superiores a los $500 \mathrm{HV}$ en espesores de $0,15 \mathrm{~mm}$.

Palabras claves: Endurecimiento superficial. Transformaciones de dureza en estado sólido. Tratamiento térmico con láser.

\section{Surface heat treatment of U8 steel by Nd:YAG laser}

\begin{abstract}
The feasibility of laser surface hardening of U8 steel using a Nd:YAG continuous wave laser has been shown in this work. With the present trade literature reviewed and the experimental procedure we developed an outline of the process parameters determination methodology. It is found that in spite of the limitations and over-simplification of the experiment, an average explanation of the observed phenomena can be made to illustrate the parameters operation limits. The operation limits of the three principal parameters when hardening U8 steel are described, and it is shown that the depth of hardening is closed correlated with the incident power on the specimen $(P)$, the diameter of the laser beam $(d)$ and the scan velocity by $P / \sqrt{d v}$. The experiment was arranged statistically and the surface hardness of U8 steel can be increased to values above $500 \mathrm{HV}$ in a deep of $0.15 \mathrm{~mm}$.
\end{abstract}

Keywords: Surface hardening. Solid state transformation hardening. Laser heat treating.

\section{INTRODUCCIÓN}

El rayo láser es un haz luminoso de alta intensidad y direccionalidad, su empleo como instrumento tecnológico esta basado en la interacción de la luz con la materia. El tratamiento con láser es una forma efectiva de variar la estructura, composición y las propiedades de capas superficiales de materiales, fundamentalmente metálicos. La modificación de propiedades superficiales en piezas metálicas tiene gran importancia si tenemos en cuenta que los nuevos materiales metálicos necesarios para el desarrollo tecnológico son un compromiso entre las

(*) Grupo Tribológico. Univ. de Oriente. Fac. de Ingeniería Mecánica. Ave. de las Américas, s/n CP 90900 Santiago de Cuba (Cuba).

(**) Grupo TECNOLÁSER. Dpto. de Física. ISPJAE. Ciudad Habana (Cuba) características generales del material y las específicas de su superficie ( 1 y 2 ).

En la figura 1 se muestra un esquema del proceso tecnológico del tratamiento térmico superficial con láser (TTSL).

El haz del láser es enfocado a la superficie del metal mediante un sistema óptico. El barrido del haz sobre la superficie metálica permite obtener un trazo sobre la misma. Los parámetros de irradiación durante el proceso son controlados para evitar el fundido de la superficie ya que en lo referente a esta técnica se logran transformaciones de dureza en estado sólido. La zona afectada por el calor (ZAC) es la zona donde ocurren estas transformaciones a determinada profundidad (Fig. 2).

Los parámetros tecnológicos más importantes en el proceso: potencia incidente $P$, diámetro del haz $d$, velocidad de barrido $v$, propiedades termofísicas 


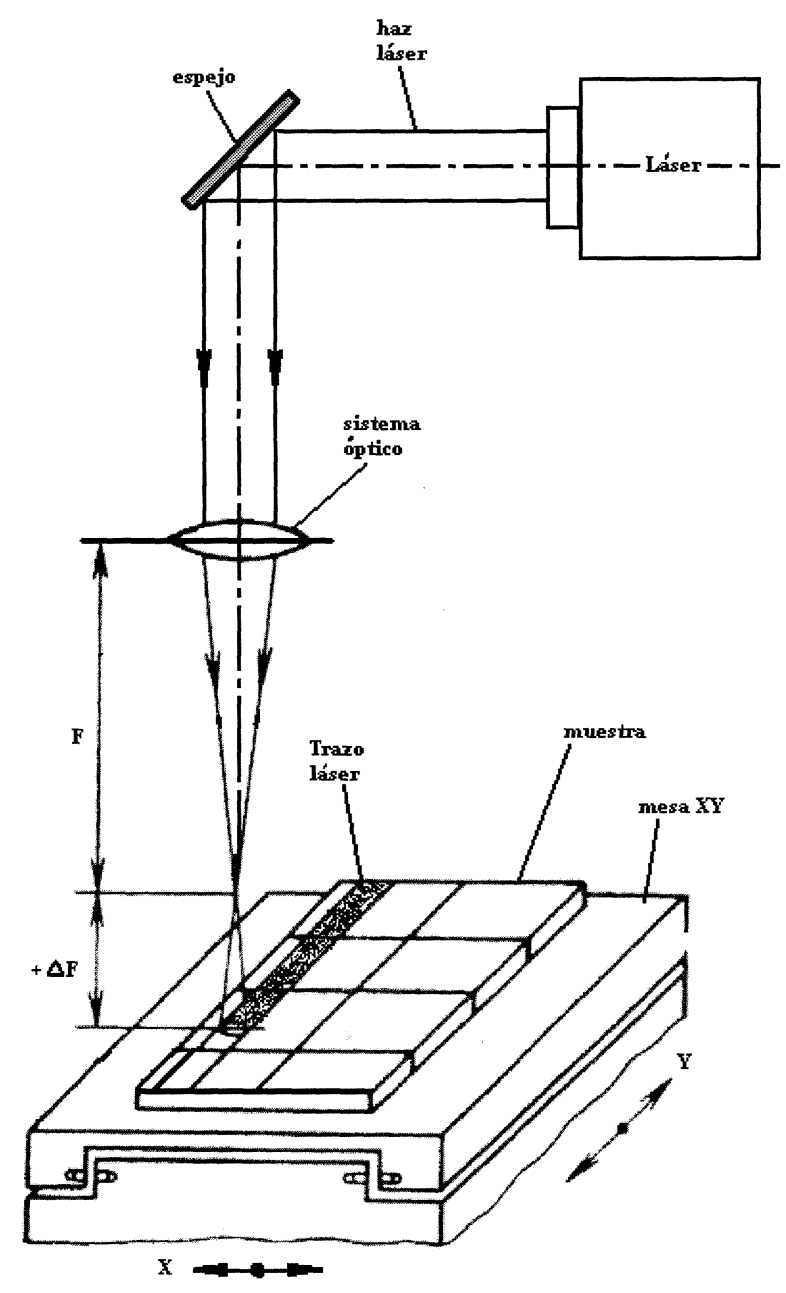

FIG. 1.- Instalación experimental.

FIG. 1.-Experimental device.

del substrato, absorción superficial de energía, determinan la profundidad endurecida y su microdureza. A partir de $500 \mathrm{~W} / \mathrm{cm}^{2}$ se obtienen gradientes térmicos en superficie elevados, al ser imposible la liberación de toda la energía absorbida por conducción al interior del material. La máxima temperatura superficial permitida es la de fusión del material, por tanto en el proceso de TTSL debe mantenerse por debajo de la misma. De este modo la temperatura de templado y los necesarios porcentajes de enfriamiento, imponen un límite a la profundidad endurecida sobre la base de la potencia disponible (3).

\section{PROCEDIMIENTO EXPERIMENTAL}

Se ha utilizado una instalación láser LTI 702, acoplada a una mesa $X Y$ y mediante una óptica de enfoque, el haz incide perpendicularmente sobre la muestra, variando el diámetro del spot por medio

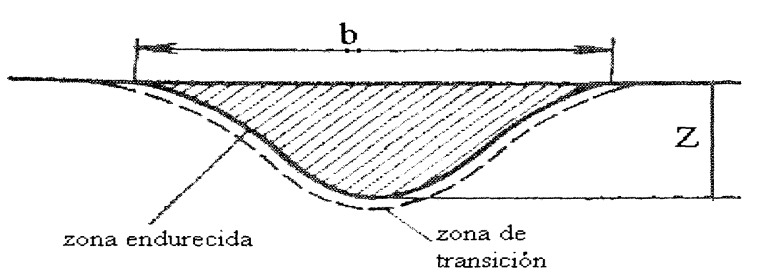

FIG. 2.- Corte transversal de una ZAC.

FIG. 2.- Transversal section of a HAZ.

del desenfoque de éste. La tabla I recoge las especificaciones del láser.

Las dimensiones de las probetas de acero U8 utilizadas en el trabajo fueron $40 \times 10 \times 5 \mathrm{~mm}^{3}$, ver (3).

La metodología del trabajo se basó en el aprovechamiento máximo de la potencia, dada las potencias bajas, y para un tamaño del spot determinado, se modificó la velocidad del proceso. La reflectividad superficial y las propiedades térmicas del substrato no serán tomadas en cuenta, ya que la primera de estas es totalmente constante al aplicarle el recubrimiento de negro de hollín a la superficie en una capa $0.01 \mathrm{~mm}$, y la segunda queda aproximadamente constante ya que las probetas son de un mismo material e igual volumen, por lo tanto las tres primeras variables mencionadas arriba serán las que guardarán relación con las variables dependientes, las cuales son: i) Profundidad de endurecimiento. ii) Ancho de la zona afectada por el haz. iii) Microestructura del ZAC.

El experimento fue ordenado como un arreglo factorial de tres factores con dos niveles cada uno. Potencia: Se utilizó un potenciómetro Ealing con una exactitud $0,1 \mathrm{~W}$, fijando $78 \mathrm{~W}$ y $68 \mathrm{~W}$. Diámetro del haz: Esta variable es la más compleja de medir, se reportan varios métodos en (4), el método utilizado es el quemado de papel (5). Velocidad de barrido: Se utilizó una mesa $X Y$, automatizada en la cual las velocidades están prefijadas $(0.02,0.06$, $0.625,2.5 \mathrm{~mm} / \mathrm{s}$ ), desechando la primera de éstas ya que se obtiene el fundido del material. Las velocidades fueron: $0.625 \mathrm{~mm} / \mathrm{s}$ y $2.5 \mathrm{~mm} / \mathrm{s}$.

TABLA I.- Parámetros del equipo láser LTI 702.

TABLE I.-Laser device parameters of LTI 702.

\begin{tabular}{|l|l|}
\hline Longitud de onda: $1,060 \mathrm{~nm}$ & Potencia de salida garantizada: $90 \mathrm{~W}$ \\
\hline Diámetro: $3 \mathrm{~mm}$ & Rango de potencia: $0-120 \mathrm{~W}$ \\
\hline Divergencia: $2 \times 10^{-3} \mathrm{rad}$ & Régimen de trabajo: continuo $(\mathrm{CW})$ \\
\hline${\text { Modo: } \text { TEM }_{01}}$ & \\
\hline
\end{tabular}

Rev. Metal. Madrid, 34 (2), 1998 
TABLA II.- Composición química del acero U8.

TABLE II.-Chemical composition of U8 steel.

\begin{tabular}{|c|c|c|c|c|c|c|c|} 
Elementos & $\mathrm{C}$ & $\mathrm{Mn}$ & $\mathrm{Cr}$ & $\mathrm{Ni}$ & $\mathrm{Ti}$ & $\mathrm{T} . \mathrm{a}$. & Dureza \\
\hline Contenido \% & 0,79 & 0,22 & 0,15 & - & - & $722^{\circ} \mathrm{C}$ & $198-207 \mathrm{HV}$ \\
\hline
\end{tabular}

\section{RESULTADOS Y DISCUSIÓN}

$\mathrm{Al}$ procesar los resultados de los experimentos realizados (Tabla III) mediante análisis de regresión lineal, se obtiene un coeficiente de correlación lineal entre la profundidad endurecida y $P / \sqrt{d v}$ de 0,8137 que comparado con el reportado por Steen $r=0,8812$ en (1), vemos que están bastante próximos, obteniéndose así un adecuado ajuste lineal con ecuación de la recta $P E=2,13 \cdot 10^{-3} P / \sqrt{d v}+$ $7,81 \cdot 10^{-2}(3)$.

Al realizar la comparación del ajuste de estos dos factores podemos llegar a la conclusión de que el primero de éstos es el más conveniente, resultado que podía ser esperado por tener este factor relacionadas las tres variables independientes. Esto coincide con lo reportado por Steen (6) y no con lo reportado por Shang (7), el cual solamente toma en cuenta en su factor dos variables.

Las mediciones realizadas de microdureza se exponen en las figuras 3 y 4 . De su análisis podemos observar la influencia de las diferentes variables en la profundidad endurecida, al mantener las otras dos constantes. Llegando a los siguientes resultados; la velocidad al mantener $P$ y $d$ constante, influye en la profundidad endurecida de forma lineal y pendiente negativa, quiere decir que al aumentar la velocidad disminuye la profundidad de endurecimiento, esto es debido a que también disminuye el tiempo de interacción y no se logra un gradiente térmico lo suficientemente pequeño para que la temperatura fluya hasta grandes profundidades. Su-

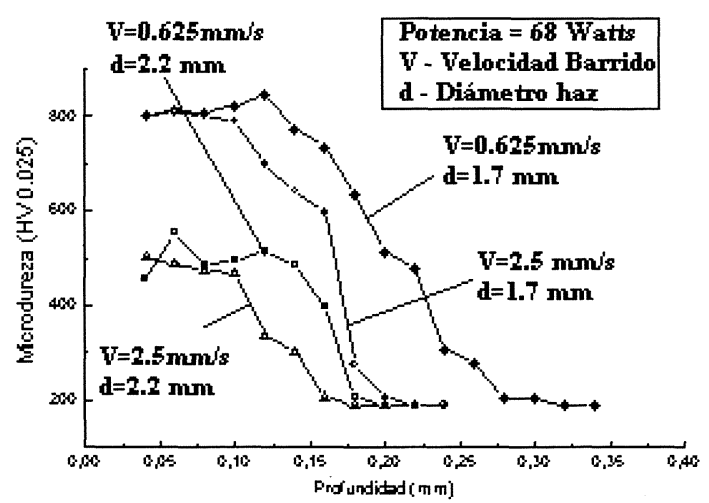

Fig. 3.- Perfil de microdurezas. Influencia de los parámetros principales.

FIG. 3.-Microhardness profiles. Influence of main parameters.

cediendo igual con el diámetro dejando fijas $P$ y $v$, al aumentar éste disminuye la profundidad endurecida. Ocurriendo lo contrario con la potencia ya que al dejar fijas $d$ y $v$, al aumentar la potencia aumenta la densidad de potencia en la superficie, manteniéndose igual el tiempo de interacción y así aumentará la profundidad endurecida hasta el punto de fundido, aspecto indeseable en este trabajo.

De estas gráficas se obtiene un resultado muy importante que es la dureza que se logra en la zona afectada por el calor, que en nuestro caso esta por encima de los $500 \mathrm{HV}$ a una profundidad superior a los 0,15 $\mathrm{mm}$ (ver figuras). En estas zonas de alta dureza, después de las observaciones realizadas en el microscopio se comprobó la existencia de la estructura martensítica de diferentes tipos, granos gruesos en un caso y en otros granos finos como agujas o pajas; observando casos en que existían los

TABLA III.- Parámetros y resultados del experimento.

TABLE III.-Experiment results and parameters.

\begin{tabular}{|c|c|c|c|c|c|c|}
\hline & PE (mm) & Pot. (W) & Vel. (mm/s) & Diam. (mm) & & \\
\hline 1 & 0,26 & 78 & 0,625 & 1,7 & 75,6711 & 98,6631 \\
2 & 0,18 & 78 & 2,500 & 1,7 & 37,8356 & 49,3315 \\
3 & 0,23 & 78 & 0,625 & 2,2 & 66,5186 & 98,6631 \\
4 & 0,19 & 78 & 2,500 & 2,2 & 33,2593 & 49,3315 \\
5 & 0,20 & 68 & 0,625 & 1,7 & 65,9697 & 86,0140 \\
6 & 0,15 & 68 & 2,500 & 1,7 & 32,9848 & 43,0070 \\
7 & 0,17 & 68 & 0,625 & 2,2 & 57,9906 & 86,0140 \\
8 & 0,10 & 68 & 2,500 & 2,2 & 28,9953 & 43,0070 \\
\hline
\end{tabular}




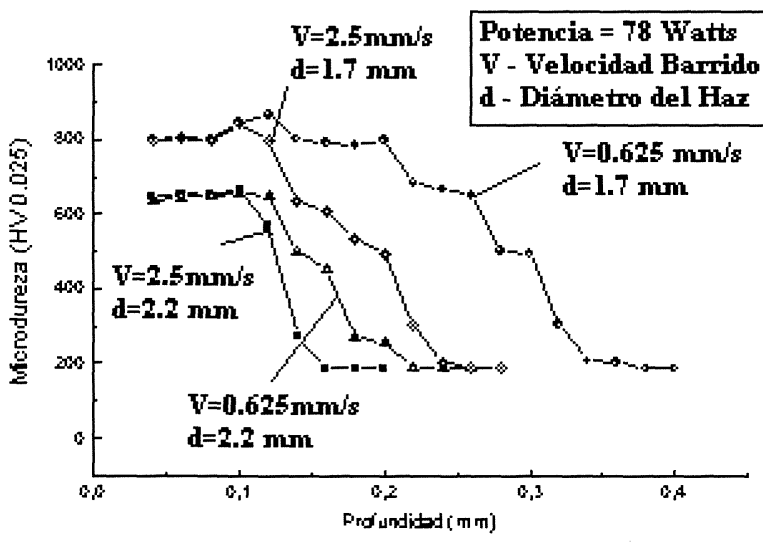

FIG. 4.- Perfil de microdurezas. Influencia de los parámetros principales.

FIG. 4.- Microhardness profiles. Influence of the main parameters.

tipos de martensita responsables de esta variación de la dureza.

\section{CONCLUSIONES}

Se consiguió un endurecimiento superficial del acero U8 mediante láser de Nd:YAG, con potencias de $78 \mathrm{~W}$ y $68 \mathrm{~W}$, con durezas superiores a $500 \mathrm{HV}$ a profundidades de $0,15 \mathrm{~mm}$. La profundidad endurecida depende de los tres parámetros básicos de irradiación: potencia, diámetro y velocidad. Siendo esta dependencia lineal con el factor que los relaciona $P / \sqrt{d v}$ y lineal también con un parámetro que se varíe manteniendo fijo los restantes. Para este acero y el láser mencionado los rangos de va- riación de los parámetros que garantizan mayor eficiencia en el endurecimiento térmico superficial son: la mayor potencia utilizada, diámetro entre 1,7 y $2,2 \mathrm{~mm}$, y velocidad entre 0,625 y $2,5 \mathrm{~mm} / \mathrm{s}$. La reproducibilidad de los resultados fue pobre, se considera relacionado con la dificultad de medir el diámetro del haz y la poca estabilidad en potencia que presentó el generador.

\section{Agradecimiento}

Los autores del trabajo desean agradecer a los organizadores de la Lista Láser (Red Iris, CSIC) y a la Red Iberoamericana de Procesamiento de Materiales por Láser (CYTED), por la ayuda suministrada. Al Departamento de Física del ISPJAE e integrantes del Grupo TECNOLASER por la ayuda en la parte inicial del trabajo.

\section{REFERENCIAS}

(1) Damborenea, J.J. DE, en Corrosión y Protección Metálicas. Vol II. Cap. 17, Avances en sistemas de protección anticorrosivos: tratamientos de modificación superficial, editores M.C. Andrade, S. Feliu. CSIC, Madrid 1991.

(2) Damborenea, J.J. DE, Dorronsoro, M., Lopez, V. y VazQUEZ, A.J. Rev. Metal. Madrid 26 (1990) 247-253.

(3) Barja Gonzalez, B. y Torres Pupo, C. Tésis de Grado. Departamento de Física, Universidad de Oriente, Jul., 1997.

(4) Stenn, W.M. y Courtney, C. Appl, Phys., 17, 1978: 303307.

(5) Gonsalves, J.N. y Duley, W.W. Can. J. Phys., 49, 1971: 1708-1713.

(6) Steen, W.M. y Courtney, C. Metals Tech., Dic., 1979: 456-462.

(7) Shang, H.M. J. Mater. Process. Technol., 23, 1990: 119. 